\title{
The MINOS Scintillator Calorimeter System
}

P. Adamson, K. V. Alexandrov, G. Alexeev, T. Alexopoulos, W. W. M. Allison, G. J. Alner, B. Anderson,

D. F. Anderson, K. Anderson, C. Andreopoulos, C. Arroyo, D. Attree, F. Avignone, D. S. Ayres, B. Baller,

B. Barsh, M. Barker, P. D. Barnes, Jr., W. L. Barrett, R. H. Bernstien, S. Bilenky, G. J. Bock, D. J. Boehnlein, D. Bogert, P. M. Border, C. Bower, E. Buckley-Geer, A. Byon-Wagner, T. R. Chase, S. Chernichenko, S. Childress,

B. C. Choudhary, J. H. Cobb, A. Cohee, J. D. Cossairt, H. Courant, J. W. Dawson, D. DeMuth, P. J. Dervan,

A. DeSanto, N. Diaczenko, M. V. Diwan, G. Drake, M. Drew, R. Ducar, T. Durkin, R. Edgecock, S. Eichblatt,

A. Erwin, E. Falk Harris, G. J. Feldman, N. Felt, T. H. Fields, H. R. Gallagher, M. Gebhard, Yu. Gilitsky,

N. Giokaris, M. C. Goodman, Yu. Gornushkin, N. Grossman, J. J. Grudzinski, V. J. Guarino, V. Gudkov, J. Guo, Yu. Gutnikov, R. Halsall, J. Hanson, D. Harris, P. G. Harris, E. Hartouni, R. Hatcher, S. Hayden, R. Heinz, K. Heller, N. Hill, Q. H. Hu, T. Hu, J. Hylen, M. Ignatenko, G. Irwin, C. James, T. Joffe-Minor, T. Kafka, S. M. S. Kasahara,

E. Katsavounidis, J. Kilmer, H. Y. Kim, V. Kochetkov, G. Koizumi, S. Kopp, M. Kordosky, D. Krakauer,

Z. Krumstein, A. Kulik, Y. F. Lai, K. Lang, C. Laughton, R. LeBeau, A. Lebedev, R. Lee, P. J. Litchfield, J. Liu,

N. P. Longley, P. Lucas, S. Madani, V. Makeev, W. A. Mann, A. Marchionni, M. L. Marshak, J. McDonald,

J. R. Meier, E. Melnikov, G. I. Merzon, M. D. Messier, D. G. Michael, R. H. Milburn, L. Miller, W. H. Miller,

S. R. Mishra, P. S. Miyagawa, J. Mofin, R. Morse, L. Mualem, S. Mufson, M. Murtagh, J. Musser, A. Napier,

D. Naples, C. Nelson, J. K. Nelson, H. Newman, T. C. Nicholls, A. Nozdrin, J. Oliver, W. Oliver, A. Olshevski, V. Onuchin, V. Paolone, A. Para, T. Patzak, G. F. Pearce, N. Pearson, C. W. Peck, C. Perry, E. A. Peterson, D. A. Petyt, A. Pla-Dalmau, R. K. Plunkett, L. E. Price, D. R. Pushka, R. A. Rameika, A. L. Read, B. Rebel, C. Rosenfeld, K. Ruddick, V. A. Ryabov, R. Saakyan, A. Sadovski, M. Sanchez, N. Saoulidou, J. Schneps, P. V. Schoessow, V. Semenov, I. Shein, B. W. Sheng, A. Sisakian, W. Smart, V. Smirnitsky, C. Smith, P. N. Smith, V. Smotriaev, A. Soldatov, N. Solomey, R. Soltz, N. I. Starkov, P. Sullivan, E. C. Swallow, M. Szigety, R. Talaga, J. Thomas, J. L. Thron, V. Tokmenin, D. N. Tovee, R. Trendler, J. Trevor, I. Trostin, V. A. Tsarev, G. Tzanakos, G. Unel, J. Urheim, A. Usachev, T. Vahle, M. M. Velasco, L. Wai, D. Wark, A. Weber, R. C. Webb, A. Wehmann, N. West, R. F. White, R. Wildberger, R. Winston, S. G. Wojcicki, D. Wright, X. M. Xia, W. G. Yan, J. C. Yun, and Z. A. Zhu

Abstract-The MINOS detectors will use extruded plastic scintillator strips, which are read out by wavelength-shifting fibers coupled to multipixel photodetectors. This technique provides excellent energy and spatial resolution. The MINOS detectors are described in detail along with results from light output tests.

Index Terms-Calorimeter, MINOS, scintillator.

\section{INTRODUCTION}

$\mathbf{T}$ HE Main Injector Neutrino Oscillation Search (MINOS) [1] experiment is designed to search for neutrino oscillations [2], [3] with a sensitivity significantly greater than has been achieved to date.

If neutrinos do oscillate, as suggested by some experimental results [4]-[6] and by theoretical extensions of the standard model of particle physics, then they will change their flavor (electron, muon, tau, or sterile) as they move through space or matter. The probability for a neutrino of flavor $\nu_{l}$ to oscillate in vacuum into one of flavor $\nu_{l^{\prime}}$ is given by (1)

$$
P\left(\nu_{l} \rightarrow \nu_{l^{\prime} \neq l}\right)=\sin ^{2} 2 \theta \sin ^{2}\left[1.27 \triangle M^{2} \frac{L}{E}\right]
$$

Manuscript received April 4, 2001. This work was supported by the U.S. Department of Energy and by the U.K. Particle Physics and Astronomy Research Council.

P. J. Dervan, representing the MINOS Collaboration, is with the Department of Physics and Astronomy, University College London, WC1 6BT London, U.K. (e-mail: pjd@hep.ucl.ac.uk).

Publisher Item Identifier S 0018-9499(02)06121-X. where $\theta$ is the mixing angle, $\Delta M$ is the difference in mass between the two flavors of neutrino, $L$ is the distance travelled until the neutrino oscillates, and $E$ is the energy of the neutrino. Hence, for neutrinos to oscillate, they must have mass, which is contrary to the standard model of particle physics, where the mass of all flavors of neutrinos is assumed to be zero. Different flavors of neutrinos (electron, muon, tau, or sterile) can be identified by distinctively different patterns of secondary particles they produce when they interact with matter in a massive neutrino detector. Thus an optimum, and most sensitive, way to detect such oscillations is to compare the patterns of their interactions (characterized by topology and energy deposition) at two widely separated locations in a neutrino beam. The MINOS experiment is based on this design, using a $\nu_{\mu}$ beam, and is most sensitive to $\nu_{\mu} \rightarrow \nu_{e}$ transitions, but will also be able to search for $\nu_{\mu} \rightarrow \nu_{\tau}$ transitions. The two MINOS detectors are located at Fermilab (near detector) and in the Soudan mine in Minnesota, $730 \mathrm{~km}$ away (far detector).

The MINOS detectors are iron-scintillator sandwich calorimeters, with toroidal magnetic fields in their thin steel planes. MINOS will use a scintillator with sufficiently fine transverse granularity, so as to provide both calorimetry and tracking information. The scintillator strips are extruded polystyrene with a $\mathrm{TiO}_{2}$ outer layer for reflectivity.

Extensive tests have been carried out on scintillator modules (20 or 28 strips wide) using cosmic rays. The average light output per minimum ionizing particle in the center of the strip is six photoelectrons. 


\section{THE MINOS EXPERIMENT}

\section{A. The NuMI Beam}

The neutrino beam [7] relies on primary $120-\mathrm{GeV}$ protons from the main injector, extracted in an 8.4-s-long single turn extraction every $2 \mathrm{~s}$. The protons are transported to the downstream target hall and aimed downwards at $3.3^{\circ}$ so as to point at the MINOS far detector. The main injector is expected to deliver about $4 \times 10^{13}$ protons perpulse on target. The target is a small diameter (matched to the transverse size of the proton beam) segmented graphite cylinder about $160 \mathrm{~cm}$ long. The resulting hadron beam is subsequently sign selected and focused by specially designed focusing elements and then transported through an evacuated decay pipe, $1 \mathrm{~m}$ in radius and $675 \mathrm{~m}$ long, before striking a secondary absorber downstream. The total decay length is $725 \mathrm{~m}$. The dolomite between the hadron absorber and the first MINOS detector provides sufficient shielding to range out all the muons produced by pion and kaon decays in the beam pipe.

\section{B. The MINOS Detectors}

The MINOS detectors consist of the near detector at Fermilab and the far detector at Soudan, $730 \mathrm{~km}$ away and $713 \mathrm{~m}$ below ground. They are iron-scintillator sandwich calorimeters. The active elements are $1-\mathrm{cm}$-thick and $4.1-\mathrm{cm}$-wide scintillator strips. The scintillator strips are extruded STYRON $663-\mathrm{W}$ polystyrene, doped with $1 \%$ of PPO and $0.03 \%$ POPOP. A $0.25-\mathrm{mm}$-thick reflective layer, obtained by adding $12.5 \%$ of $\mathrm{TiO}_{2}$ to polystyrene and coextruded with the scintillator, surrounds each strip, except along a 1.4-mm-wide and 2.0-mm-deep groove also coextruded for embedding a light-collecting 1.2-mm-diameter wavelength-shifting (WLS) fiber. The WLS fiber is a J-type Y11 multiclad polymethylmethacrylate, non-S, and doped with K27 dye at $175 \mathrm{ppm}$ (maximum intensity emission at $520 \mathrm{~nm}$ ). The light attenuation in the WLS fibers is well modeled by a double exponential falloff with $\lambda_{1} \approx 0.7 \mathrm{~m}$ and $\lambda_{2} \approx 3.9 \mathrm{~m}$. Signals from the WLS fibers are further transported by clear polystyrene Kuraray fibers with attenuation length $\lambda \approx 13 \mathrm{~m}$.

1) The Far Detector: The MINOS far detector is a 5.4 metric kt magnetized iron calorimeter consisting of two supermodules. The basic module is an 8 -m-diameter (octagonal geometry) $2.54-\mathrm{cm}$ - thick steel plane. A coil running through the steel provides a 1.5-T magnetic field. The far detector consists of 486 planes. Each scintillator plane is made up of eight modules containing 20 or 28 strips depending on their location in the plane (Fig. 1). Each plane consists of 198 strips in total. The orientation of the strips alternates by $90^{\circ}$ in successive planes.

The far detector is read out at both ends using a total of 1452 16-pixel Hamamatsu R5900-M16 photomultiplier tubes. Eight different fibers, from eight strips spaced roughly $1 \mathrm{~m}$ apart on the detector, are coupled to each pixel. The resultant eight-fold ambiguity can be resolved in software by utilizing the fact that the exact arrangement of which fiber is coupled to which pixel is somewhat different at the two strip ends. The characteristics of the M16s have been extensively tested [8]. The effective collection efficiencies and gains (at $10^{6}$ ) in individual pixels are uniform within about $10 \%$ and $15 \%$, respectively, if only the

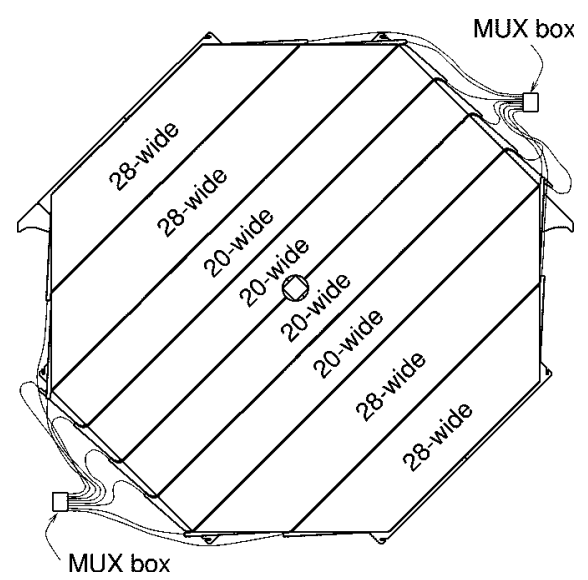

Fig. 1. Scintillator modules on one plane of the far detector. The MUX boxes contain the phototubes.

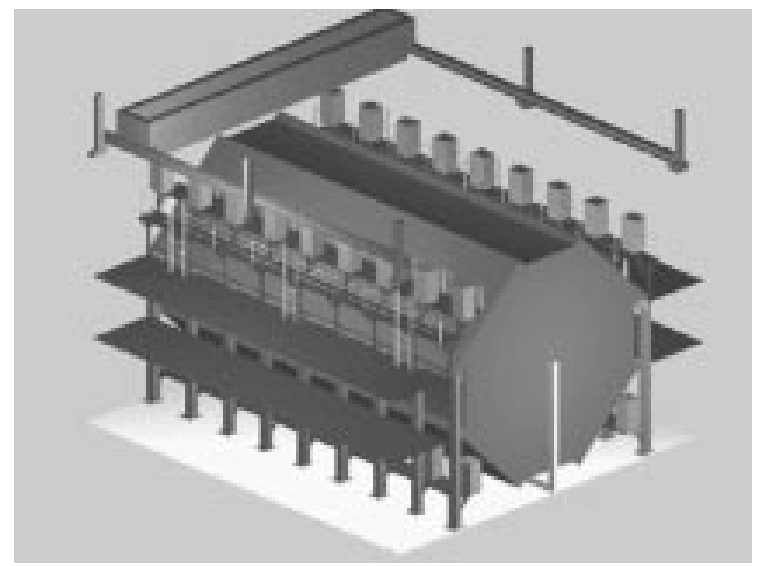

Fig. 2. Schematic view of the far detector. The boxes on the platforms at the sides of the detector are where the front-end electronics, high-voltage power supplies, and photodetectors are situated.

nominal MINOS fiber positions are considered. The crosstalk is dominated by optical spreading of photons impinging upon the tube through the 1.2-mm-diameter fiber. Near-neighbor (sidesharing) pixels pick up less than $5 \%$ signal, while diagonalneighbor pixels pick up less than $1 \%$ of the signal. In both cases, the crosstalk strongly depends on the relative position of the center of the fiber with respect to the edge of the pixel. These tests showed that these multianode tubes meet the requirements of the MINOS detectors.

Simulations have been carried out using a program called gminos. This program combines a description of the detector geometry, the neutrino flux from GNuMI [9], and the simulation of the properties of the scintillator and photodetectors with the standard GEANT [10] tracking and particle interaction program. Using the current detector design, a hadronic energy resolution of $\Delta E / E \approx 55 \% / \sqrt{E}$ and a muon momentum resolution of $\Delta p / p \approx 15 \%$ are expected [1].

Fig. 2 shows a schematic view of the far detector.

2) The Near Detector: The near detector attempts to emulate that of the far detector in all relevant properties: the nature and thickness of the absorber planes, the nature and granularity of the active detector, and the strength of the magnetic field. The basic near-detector geometry is an elongated octagon, $3.8 \mathrm{~m}$ high and $4.8 \mathrm{~m}$ wide. The coil hole is placed off center 


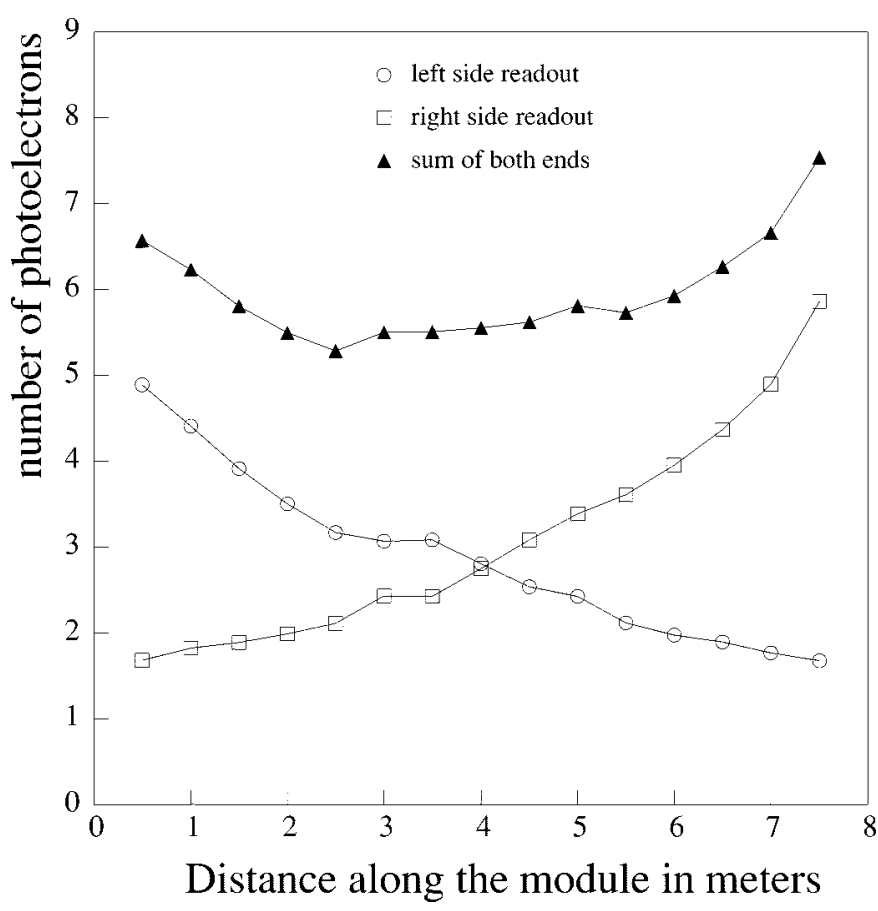

Fig. 3. Typical light yield from a full-size prototype scintillator module.

horizontally, and the detector itself is positioned so that the central part of the neutrino beam is about $1 \mathrm{~m}$ away from the hole. In the longitudinal dimension, the detector is composed of four functionally different components. Starting from the upstream end, they are as follows.

1) Veto part. Neutrino interactions in this region are not used because of possible end effects and the need to ensure that there is no background from neutron interactions. This part has a thickness of $0.5 \mathrm{~m}$ of steel.

2) Target part. Neutrino interactions in this part are used for near/far comparison. It has a thickness of $1.5 \mathrm{~m}$ of steel.

3) Hadron shower part. This part is long enough to contain the full showers of all neutrino interactions occurring in the target part. It has a thickness of $1.5 \mathrm{~m}$ of steel.

4) Muon spectrometer. This part is used to range out muons and measure their momentum. This part is $4 \mathrm{~m}$ of steel thick.

In the first three parts, all planes are instrumented, whereas only every fourth plane is instrumented in the muon spectrometer. Only the central part of the neutrino beam, $r<25 \mathrm{~cm}$, is used in the near detector to reduce near/far differences. Hence only part of the area of the upstream sections is instrumented. In total, the near detector consists of 200 planes of iron scintillator. Each strip is read out from its outer end only, onto one pixel of a Hamamatsu R5900-M64 64-pixel photomultiplier tube. The opposite ends will be reflectively terminated to boost the average light yield to a similar level as in the far detector. A total of 215 M64 tubes will be used in the near detector.

\section{LIGHT OUTPUT TESTS}

Full-size prototype scintillator modules ( 20 and 28 wide) have been tested on a cosmic-ray test stand. The light output as a function of distance along the scintillator strips (read out at both ends of the strip) was measured using cosmic rays. Fig. 3 shows the average light output for one module. The attenuation of the light can clearly be seen as the distance between the photomultiplier and the position of the cosmic-ray interaction increases. The average light output is about six photoelectrons in the center of the strips.

\section{CONCLUSION}

The light yields from full-size prototype MINOS scintillator modules have been measured and found to be $\approx 6$ photoelectrons in the middle for cosmic-ray muons. This is satisfactory for MINOS operation.

MINOS is now in its construction phase and will start taking data in 2003.

\section{REFERENCES}

[1] MINOS Collaboration, "The MINOS detectors technical design report,", Fermilab Rep. NuMI-L-337, October 1998.

[2] B. Pontecorvo, "Mesonium and anti-mesonium," Zh. Eksp. Theor. Fiz., vol. 33, pp. 549-551, 1957.

[3] S. M. Bilenky and S. T. Patcov, "Massive neutrinos and neutrino oscillations," Rev. Mod. Phys., vol. 59, p. 671, 1987.

[4] C. Athanassopoulos et al., "Evidence for $\bar{\nu}_{\mu} \rightarrow \bar{\nu}_{e}$ oscillations from the LSND experiment at LAMPF," Phys. Rev. Lett., vol. 77, pp. 3082-3085, 1997.

[5] Y. Fukuda et al., "Evidence for oscillation of atmospheric neutrinos," Phys. Rev. Lett., vol. 81, pp. 1562-1567, 1998.

[6] K. S. Hirata et al., "Real time directional measurement of B-8 solar neutrinos in the Kamiokande-II detector," Phys. Rev., vol. D44, pp. 2241-2260, 1991.

[7] "NuMI facility technical design report,", Fermilab Rep. NuMI-L-346, March 1998.

[8] K. Lang et al., "A comprehensive characterization of Hamamatsu 16and 64-anode PMT's,", Fermilab Rep. NuMI-L-688, May 2000.

[9] MINOS Collaboration, "MINOS experiment R\&D plan: FY 1996-1998,", Fermilab Rep. NuMI-L-184, July 1996.

[10] R. Brun, R. Hagelberg, M. Hansroul, and J. C. Lassalle, "GEANT: Simulation program for particle physics experiments: User guide and reference manual,", CERN Rep. CERN-DD-78-2, July 1978. 